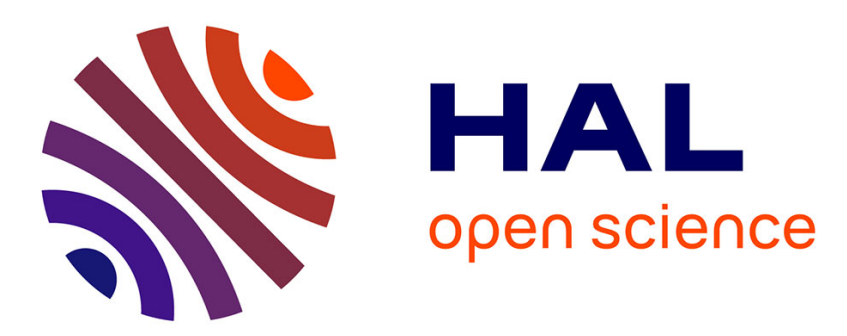

\title{
Les pucerons de Guadeloupe, des Grandes et Petites Antilles (Hemiptera, Aphididae)
}

\author{
Jean Etienne
}

\section{To cite this version:}

Jean Etienne. Les pucerons de Guadeloupe, des Grandes et Petites Antilles (Hemiptera, Aphididae).

Bulletin de la Société Entomologique de France, 2005, 110 (4-5), pp.455-462. hal-02681045

\section{HAL Id: hal-02681045 \\ https://hal.inrae.fr/hal-02681045}

Submitted on 31 May 2020

HAL is a multi-disciplinary open access archive for the deposit and dissemination of scientific research documents, whether they are published or not. The documents may come from teaching and research institutions in France or abroad, or from public or private research centers.
L'archive ouverte pluridisciplinaire HAL, est destinée au dépôt et à la diffusion de documents scientifiques de niveau recherche, publiés ou non, émanant des établissements d'enseignement et de recherche français ou étrangers, des laboratoires publics ou privés. 


\title{
Les Pucerons de Guadeloupe, des Grandes et Petites Antilles (Hemiptera, Aphididae)
}

\author{
par Jean ÉTIENNE \\ INRA, Centre Antilles-Guyane, Domaine Duclos, F - 97170 Petit-Bourg, Guadeloupe
}

Résumé. - Cette note cite 41 espèces de Pucerons pour la Guadeloupe, indique pour chacune d'entre elles leurs plantes-hôtes, leur distribution géographique et signale la présence, pour la première fois aux Antilles, de l'espèce Hyperomyzus carduellinus. Cette publication fait également le bilan de nos connaissances sur les Pucerons de la région Caraïbe et mentionne, avec quelques commentaires, les 94 espèces actuellement répertoriées dans les Grandes et Petites Antilles.

Summary. - The Aphids from Guadeloupe, the Greater and Lesser Antilles (Hemiptera, Aphididae). This note presents a list of 41 species of aphids from Guadeloupe, with their host plants, their geographic distribution and report the presence, for the first time, of Hyperomyzus carduellinus in the Caribbean. This publication also takes stock of our knowledge on the aphids of the Caribbean region with some particular comments for the 94 species currently reported in the Greater and Lesser Antilles.

Mots clés. - Hemiptera, Aphididae, liste, Guadeloupe, Grandes et Petites Antilles, région Caraïbe.

Peu d'espèces de Pucerons ont été mentionnées sur plantes en Guadeloupe. Le plus souvent les espèces répertoriées l'ont été à la faveur de recherches réalisées sur les ravageurs des cultures mais aucun travail spécifique d'inventaire n'a été effectué sur les Aphididae de cette île. C'est donc sur la Canne à sucre, culture principale de l'île, que les premiers pucerons ont été signalés: Rhopalosiphum maidis (Fitch) (BLANCHE, 1956) et Sipha flava (Forbes) (D'AGUILAR \& Bonfils, 1962). Par la suite, DelPlanQue (1976) rapporte Aphis gossypii Glover et Myzus persicae (Sulzer) sur cultures maraîchères. Dans une étude sur l'épidémiologie des virus disséminés par les Pucerons, MiGLIORI et al. (1977) citent 27 espèces d'Aphides obtenues par piégeage (pièges de Moericke). Ce premier recensement de la faune aphidienne de Guadeloupe était ignoré à l'époque par SMITH \& CERMELI (1979) qui mentionnent seulement les 4 espèces précédemment citées. LECLANT et al. révèlent en 1992 l'introduction de Toxoptera citricida (Kirkaldy), dangereux vecteur du virus de la "Tristeza" sur les agrumes.

La collecte de pucerons en Guadeloupe, bien qu'effectuée de façon non systématique, a été pratiquée de 1985 à 2003 et a permis de rassembler plus de 250 échantillons prélevés sur plantes cultivées et autres espèces végétales. Ces collectes ont permis de retrouver 20 des 27 espèces antérieurement piégées et elles ont révélé l'existence de 13 espèces supplémentaires dans l'île. Ainsi 41 espèces de Pucerons sont actuellement répertoriées de Guadeloupe. Au cours de cette même période, nous avons pu récolter des pucerons dans quelques autres îles des Caraïbes et nous en profitons pour mettre à jour les données publiées voici 26 ans par SMITH \& CERMELI (1979).

La nomenclature utilisée pour les Aphides est celle du catalogue de REMAUDIÈRE \& REMAUDIÈRE (1997); en outre, elle tient compte de la récente révision des Cerataphis inféodés aux Arecaceae, par RuSSELl (1996). La plupart des échantillons sont déposés au Muséum national d'Histoire naturelle, à Paris. La nomenclature recommandée dans la Flore de FOURNET (2002) a été retenue pour la désignation des plantes-hôtes.

Parmi les 41 espèces de Pucerons signalées ci-après, 7 ont été capturées uniquement par piégeage, tandis que 34 ont été collectées sur 85 plantes appartenant à 41 familles botaniques. L'indication des plantes-hôtes concerne seulement celles constatées en Guadeloupe, elle ne représente pas l'éventail des hôtes de ces Aphides dans le Monde. Les noms des 13 espèces 
signalées pour la première fois en Guadeloupe sont précédés d'un astérisque $\left({ }^{*}\right)$ (ou de deux, dans le cas de Hyperomyzus carduellinus qui est signalé des Antilles pour la première fois).

\section{Liste commentée des Pucerons de Guadeloupe, de leurs hôtes et de leur distribution}

Aphis coreopsidis (Thomas, 1878). Plante-hôte. - Asteraceae : Bidens alba. Cette espèce est répandue sur tout le continent américain, elle a été signalée une fois d'Afrique (Ghana) par EASTOP (1961).

Aphis craccivora Koch, 1854. Plantes-hôtes. - Anacardiaceae: Anacardium occidentale. Caesalpiniaceae : Cassia javanica var. indochinensis. Fabaceae: Cajanus cajan, Gliricidia sepium, Vigna luteola, V. unguicula. Malpighiaceae: Malpighia emarginata. Mimosaceae : Mimosa pigra. Nyctaginaceae : Bougainvillea sp. Polygonaceae: Coccoloba uvifera. Rutaceae: Citrus spp. Solanaceae: Datura sp. Cosmopolite.

Aphis gossypii Glover, 1877. Plantes-hôtes. - Acanthaceae: Justicia brandegeana. Anacardiaceae: Anacardium occidentale, Mangifera indica. Araceae: Colocasia esculenta. Asteraceae: Ageratum conyzoides, Erechtites hieracifolia. Bignoniaceae: Spathodea campanulata, Tabebuia heterophylla. Commelinaceae: Commelina diffusa. Cucurbitaceae: Cucumis melo, C. sativus, Cucurbita moschata, C. pepo. Malpighiaceae: Malpighia emarginata. Malvaceae: Abelmoschus esculentus, Hibiscus rosa-sinensis, H. sabdariffa. Mimosaceae: Calliandra purpurea. Myrtaceae: Psidium guajava. Nyctaginaceae: Bougainvillea sp. Poaceae: Zea mays. Rubiaceae: Ixora. Rutaceae: Citrus spp. Scrophulariaceae: Capraria biflora. Solanaceae: Capsicum annuum, C. frutescens, Solanum melongena, S. racemosum. Espèce cosmopolite.

*Aphis illinoisensis Shimer, 1866. Plante-hôte. - Vitaceae: Vitis vinifera. Espèce présente du nord au sud de l'Amérique, récemment introduite en Turquie (REMAudiėre et al., 2003).

Aphis nerii Boyer de Fonscolombe, 1841. Plantes-hôtes. - Apocynaceae: Calotropis procera, Nerium oleander. Asclepiadaceae: Asclepias curassavica, Metastelma parviflorum. Espèce cosmopolite.

Aphis spiraecola Patch, 1914. Plantes-hôtes. - Anacardiaceae: Mangifera indica. Asteraceae: Ageratum conyzoides, Bidens alba, Erechtites hieracifolia, Mikania micrantha. Bignoniaceae: Tabebuia heterophylla. Caesalpiniaceae: Cassia javanica var. indochinensis. Meliaceae: Lansium domesticum. Mimosaceae: Calliandra purpurea. Nyctaginaceae: Bougainvillea sp. Passifloraceae: Passiflora edulis. Rutaceae: Citrus spp., Murraya exotica. Espèce récemment devenue cosmopolite. Elle a été longtemps désignée par erreur sous le nom de $A$. citricola van der Goot (EASTOP \& BLACKMAN, 1988).

Aulacorthum solani (Kaltenbach, 1843). Plantes-hôtes. - Asteraceae: Ageratum conyzoides. Solanaceae: Solanum americanum. Espèce cosmopolite d'origine paléarctique.

Brachycaudus helichrysi (Kaltenbach, 1843). Plante-hôte. - Asteraceae : Erechtites hieracifolia. Espèce cosmopolite sur ses hôtes secondaires.

Capitophorus hippophaes (Walker, 1852). Piégeage: MIGLIORI et al. (1977). Sur son hôte secondaire, Polygonum, l'espèce est cosmopolite.

*Carolinaia cyperi Ainslie, 1915. Plante-hôte. - Cyperaceae: Cyperus rotundus. Espèce largement répandue en région néotropicale.

Cavariella aegopodii (Scopoli, 1763). Plante-hôte. - Apiaceae: Petroselinum crispum. Espèce cosmopolite sur ses hôtes secondaires, d'origine holarctique.

*Cerataphis brasiliensis (Hempel, 1901). Plantes-hôtes. - Arecaceae : Caryota urens, Chrysalidocarpus lutescens, Cocos nucifera. Zingiberaceae: Alpinia purpurata. Pantropicale originaire du Sud-Est asiatique.

*Cerataphis orchidearum (Westwood, 1879). Plante-hôte. - Orchidaceae: Vanilla planifolia. Pantropicale.

Geopemphigus floccosus (Moreira, 1925). Plantes-hôtes. - Asteraceae: Synedrella nodiflora. Dioscoreaceae : Dioscorea trifida. Espèce américaine répandue depuis la Floride (USA) jusqu'en Amérique du Sud.

${ }^{k * H y p e r o m y z u s ~ c a r d u e l l i n u s}$ (Theobald, 1915). Plante-hôte. - Asteraceae: Sonchus asper. Espèce probablement d'origine asiatique, présente de l'Afghanistan au Japon, connue d'Afrique du Nord (Algérie), d'Afrique sud saharienne, d'Amérique du Sud, d'Australie (NAUMANN-ETIENNE \& REMAUDIĖRE, 1995) et trouvée récemment en Floride (USA) (HALBERT et al., 2000), non observée en Europe ? Cet Aphide est nouveau pour les Antilles.

Hyperomyzus lactucae (Linné, 1758). Plantes-hôtes. - Asteraceae: Erechtites hieracifolia, Sonchus asper. Puceron d'origine paléarctique, aujourd'hui presque cosmopolite sur ses hôtes secondaires.

${ }^{*}$ Hysteroneura setariae (Thomas, 1878). Plantes-hôtes. - Poaceae: Digitaria decumbens, Echinochloa colona, Eleusine indica. Pantropicale originaire d'Amérique du Nord, occasionnellement observée en Europe. 
Lipaphis erysimi (Kaltenbach, 1843). Plantes-hôtes. - Brassicaceae: Brassica oleracea, Brassica rapa, Raphanus sativus. Cosmopolite.

*Macrosiphoniella sanborni (Gillette, 1908). Plante-hôte. - Asteraceae : Dendranthema indicum. Cosmopolite.

Macrosiphum euphorbiae (Thomas, 1878). Plante-hôte. - Solanaceae: Lycopersicon esculentum. Espèce cosmopolite probablement d'origine néarctique.

${ }^{*}$ Melanaphis sacchari (Zehntner, 1897). Plante-hôte. - Poaceae: Saccharum officinarum. Espèce probablement originaire d'Extrême-Orient, devenue pantropicale.

Microparsus (Picturaphis) brasiliensis (Moreira, 1925). Plante-hôte. - Fabaceae : Phaseolus lunatus. Connu d'Amérique du Sud, d'Amérique Centrale et des Caraïbes, cet aphide est également cité de Floride (USA).

Myzus ornatus Laing, 1932. Plante hôte: Asteraceae: Erechtites hieracifolia. Cosmopolite.

Myzus persicae (Sulzer, 1776). Plantes-hôtes. - Apiaceae: Apium graveolens. Asteraceae: Ageratum conyzoides, Erechtites hieracifolia. Brassicaceae: Raphanus sativus. Portulacaceae: Portulaca oleracea. Solanaceae: Capsicum annuum, Solanum melongena, Solanum tuberosum. Cosmopolite.

Pentalonia nigronervosa Coquerel, 1859. Plantes-hôtes. - Araceae: Dieffenbachia sp. Heliconiaceae : Heliconia sp. Musaceae: Musa sp. Zingiberaceae: Alpinia purpurata. Espèce pantropicale.

*Pseudoregma panicola (Takahashi, 1921). Plante-hôte. - Poaceae: Ichnanthus pallens. Cette espèce d'origine orientale a une large répartition pantropicale.

*Rhodobium porosum (Sanderson, 1900). Plante-hôte. - Rosaceae : Rosa sp. Aphide presque cosmopolite:

Rhopalosiphum maidis (Fitch, 1856). Plantes-hôtes. - Poaceae: Sorghum bicolor, Zea mays, Echinochloa colona. Espèce cosmopolite originaire d'Asie: son hôte primaire a été récemment découvert au Pakistan (REMAUDIĖRE \& NAUMANN-ETIENNE, 1991).

*Rhopalosiphum nymphaeae (Linné, 1761). Plante-hôte. - Nymphaeaceae: Nymphaea sp. Espèce d'origine paléarctique, aujourd'hui cosmopolite.

Rhopalosiphum padi (Linné, 1758). Piégeage: MigLIORI et al. (1977). Comme la précédente, cette espèce d'origine paléarctique est devenue cosmopolite.

Rhopalosiphum rufiabdominale (Sasaki, 1899). Piégeage: MigLIORI et al. (1977). Probablement originaire d'Extrême-Orient', largement rëpandue sur racines de Poaceae dans le monde entier.

Schizaphis graminum (Rondani, 1847). Piégeage: MIGLIORI et al. (1977). Espèce d'origine paléarctique, subcosmopolite, inféodée aux Poaceae, nuisible aux céréales.

Sipha flava (Forbes, 1884). Plantes-hôtes. - Poaceae: Digitaria decumbens, Saccharum officinarum. Présente sur tout le continent américain.

Sitobion avenae (Fabricius, 1775). Piégeage: MIGLIORI et al. (1977). Espèce subcosmopolite sur Poaceae, absente d'Extrême-Orient.

*Sitobion mesosphaeri (Tissot, 1934). Plante-hôte. - Lamiaceae: Hyptis verticillata. Décrite de Floride, cette espèce a été signalée de Puerto Rico par SMITH et al. (1963) puis traitée comme synonyme de Macrosiphum salviae Barthomolew, 1932, par SMITH et al. (1971). En 1997, REMAUDIĖRE \& REMAUDIĖRE rétablissent $S$. mesosphaeri comme espèce valide avec, pour synonyme, Macrosiphum hyptidis E.E. Blanchard, 1944, décrit d'Argentine. En conclusion, S. mesosphaeri est actuellement connu de Floride, de Puerto Rico, de Guadeloupe et d'Argentine.

Tetraneura nigriabdominalis (Sasaki, 1899). Plante-hôte. - Poaceae: Paspalum conjugatum. Pratiquement cosmopolite sur racines de Poaceae:

Toxoptera aurantii (Boyer de Fonscolombe, 1841). Plantes-hôtes. - Anacardiaceae : Mangifera indica. Annonaceae: Annona muricata. Clusiaceae: Mammea americana. Mimosaceae: Calliandra purpurea. Rutaceae : Citrus spp. Sterculiaceae: Theobroma cacao. Espèce subcosmopolite.

Toxoptera citricida (Kirkaldy, 1907). Plantes-hôtes. - Clusiaceae: Mammea americana. Rutaceae : Citrus spp., Murraya exotica, Zanthoxylum microcarpum. Espèce originaire d'Extrême-Orient devenue commune en Asie, en Afrique subsaharienne et, depuis 1930, en Amérique du Sud; citée de Puerto Rico en 1975 (présence très douteuse à cette date ?) par MARTORELL puis de Trinidad par SMITH \& CERMELI (1979) avant l'invasion générale des Caraïbes en 1992-1993, arrivée en Floride en 1995 (HALBERT \& BROWN, 1996).

*Uroleucon ambrosiae (Thomas, 1878). Plantes-hôtes. - Asteraceae : Ageratum conyzoides, Bidens pilosa, Erechtites hieracifolia, Mikania micrantha, Parthenium hysterophorus, Pluchea carolinensis. Espèce néarctique. 
Uroleucon erigeronense (Thomas, 1878). Piégeage: MIGLIORI et al. (1977). Espèce d'origine néarctique inféodée aux Asteraceae du genre Conyza, en expansion continue en Europe et en Asie.

Utamphorophora commelinensis (Smith, 1960). Piégeage: MIGLIORI et al. (1977). Espèce néotropicale vivant sur Commelinaceae.

Parmi les 41 espèces d'Aphides trouvées en Guadeloupe, $59 \%$ ont une distribution cosmopolite, $17 \%$ sont pantropicales et $24 \%$ sont limitées au continent américain. Sous le climat des Antilles, toutes ces espèces ont un comportement anholocyclique, sans reproduction sexuée; les hôtes primaires des espèces hétéroéciques sont absents de la région.

Compte tenu de la richesse de la flore guadeloupéenne (FOURNET, 2002) et de la variété des biotopes, il est probable qu'une intensification des prospections permettra de détecter d'autres espèces de Pucerons notamment, dans les zones d'altitude. À la faveur du développement de la navigation de plaisance, des liaisons aériennes, et de l'importation croissante de plantes exotiques ornementales, il faut également s'attendre à l'arrivée d'espèces déjà présentes dans d'autres îles antillaises ou même provenant d'autres régions du monde.

\section{Bilan des connaissances sur la faune aphidienne des Antilles}

On peut estimer à 94 le nombre total d'espèces de Puceron recensées dans les Caraïbes. Parmi ces dernières, 30 sont connues d'une seule île (Cuba), 27 sont présentes dans 2 ou 3 îles et 26 seulement dans plus de 3 îles.

Établis sur la base des informations disponibles sur les Grandes et les Petites Antilles, les tableaux I et II rassemblent les données accessibles sur la faune aphidienne de 20 îles: le tableau I concerne les neuf îles dans lesquelles plus de 7 espèces de pucerons sont recensées, tandis que le tableau II traite des onze îles dont seulement 1 à 5 espèces ont été signalées jusqu'à maintenant.

Avec 82 espèces, dont la plupart sont traitées dans la monographie de HolmaN (1974), Cuba s'avère l'île la plus riche et la mieux explorée grâce aux prospections réalisées par cet auteur entre 1965 et 1967. La faune de Puerto Rico a également été l'objet de recherches intensives qui ont été conclues par les publications de SMITH (1960 b, 1970), SMITH et al. (1963 et 1971) puis de MARTORELL (1975); depuis l'arrivée de Toxoptera citricida en 1992, on dénombre 56 espèces dans cette île. La Guadeloupe, avec les 41 espèces présentées dans le chapitre précédent, se place au troisième rang des îles des Caraïbes dont les Aphides ont été réellement explorés. Dans l'île Hispaniola (= Haïti + République Dominicaine), 24 espèces ont été reconnues, 10 d'entre elles citées par SMITH \& CERMELI (1979) et 14 supplémentaires signalées par SCHMUTTERER (1990). Dans la Jamaïque, 14 espèces sont indiquées par SMITH \& CERMELI (1979), auxquelles il faut ajouter Toxoptera citricida arrivé en 1992 ou 1993 (ÉTIENNE et al., 1993). En Martinique, on connaît 14 espèces, six sont précisées par RYCKEWAERT (1998), deux autres ont été récemment observées (Aphis nerii et Cerataphis brasiliensis). De la Barbade, 10 espèces ont été citées par BENNETT \& ALAM (1985). Dix espèces ont été trouvées à Marie-Galante, toutes déjà recensées en Guadeloupe toute proche. Parmi les 8 espèces mentionnées à Trinidad par SMITH \& CERMELI en 1979, on note déjà Toxoptera citricida, treize ans avant la brutale invasion des Antilles par cet Aphide; sa présence à cette date n'est toutefois pas surprenante car Trinidad est seulement à une quinzaine de kilomètres des côtes vénézuéliennes ; or l'espèce était déjà implantée en Amérique du Sud depuis 50 ans.

Le tableau II traite des onze îles d'où sont globalement citées une dizaine d'espèces pantropicales et cosmopolites dans les articles de FENNAH (1947), WALKER et al. (1973), ÉTIENNE et al. (1992, 1994), ÉTIENNE (1997) et WoODRUFF et al. (1998).

Parmi les 94 espèces aphidiennes reconnues dans les Antilles, une dizaine ont été originellement décrites de cet archipel. Cette situation ne signifie pas l'existence de pucerons endémiques dans cette zone. Une des 5 espèces décrites de Cuba, Aphis amaranthi Holman, 1974, 
Tableau I. - Distribution des Aphides recensés dans les 9 îles antillaises où plus de 7 espèces ont été reconnues. $\mathrm{x}$ : espèces déjà citées dans la littérature; $\mathrm{xx}$ : espèces nouvellement observées; Cu.: Cuba; Ja.: Jamaïque; Hi.: Hispaniola; P.R. : Puerto Rico; Gu. : Guadeloupe, M.G.: Marie-Galante; Ma.: Martinique; Bar.: Barbade; Tr.: Trinidad.

\begin{tabular}{|c|c|c|c|c|c|c|c|c|c|}
\hline Espèces & $\mathrm{Cu}$. & Ja. & Hi. & P.R. & Gu. & MG. & Ma. & Bar. & Tr. \\
\hline Acyrthosiphon bidenticola Smith, 1960 & $\mathrm{x}$ & & & $\mathrm{x}$ & & & & & \\
\hline Acyrthosiphon pisum (Harris, 1776) & $\mathrm{x}$ & & & & & & & & \\
\hline Aphis amaranthi Holman, 1974 & $\mathrm{x}$ & & & & & & & & \\
\hline Aphis coreopsidis (Thomas, 1878) & $\mathrm{x}$ & & & $\mathrm{x}$ & $\mathrm{x}$ & & & & \\
\hline Aphis craccivora Koch, 1854 & $\mathrm{x}$ & $\mathrm{x}$ & $\mathrm{x}$ & $\mathrm{x}$ & $\mathrm{x}$ & $\mathrm{xx}$ & $\mathrm{x}$ & $\mathrm{x}$ & $\mathrm{x}$ \\
\hline Aphis fabae Scopoli, 1763 & & & $\mathrm{x}$ & $\mathrm{x}$ & & & & & \\
\hline Aphis forbesi Weed, 1889 & $\mathrm{x}$ & & & & & & & & \\
\hline Aphis gossypii Glover, 1877 & $\mathrm{x}$ & $\mathrm{x}$ & $\mathrm{x}$ & $\mathrm{x}$ & $\mathrm{x}$ & $\mathrm{xx}$ & $\mathrm{x}$ & $\mathrm{x}$ & $\mathrm{x}$ \\
\hline Aphis helianthi Monell in Riley \& Monell, 1879 & $\mathrm{x}$ & & & & & & & & \\
\hline Aphis illinoisensis Shimer, 1866 & $\mathrm{x}$ & $\mathrm{x}$ & $\mathrm{x}$ & $\mathrm{x}$ & $\mathrm{xx}$ & & & & \\
\hline Aphis middletonii Thomas, 1879 & $\mathrm{x}$ & & & & & & & & \\
\hline Aphis nerii Boyer de Fonscolombe, 1841 & $\mathrm{x}$ & $\mathrm{x}$ & $\mathrm{x}$ & $\mathrm{x}$ & $\mathrm{x}$ & $\mathrm{xx}$ & $\mathrm{xx}$ & $\mathrm{x}$ & $\mathrm{x}$ \\
\hline Aphis spiraecola Patch, 1914 & $\mathrm{x}$ & $\mathrm{x}$ & $\mathrm{x}$ & $\mathrm{x}$ & $\mathrm{x}$ & $\mathrm{xx}$ & $\mathrm{x}$ & $\mathrm{x}$ & $\mathrm{x}$ \\
\hline Aulacorthum circumflexum (Buckton, 1876) & $\mathrm{x}$ & & & $\mathrm{x}$ & & & & & \\
\hline Aulacorthum solani (Kaltenbach, 1843) & $\mathrm{x}$ & & $\mathrm{x}$ & $\mathrm{x}$ & $\mathrm{x}$ & & $\mathrm{x}$ & & \\
\hline Brachycaudus prunicola (Kaltenbach, 1843) & $\mathrm{x}$ & & & & & & & & \\
\hline Brachycaudus helichrysi (Kaltenbach, 1843) & $\mathrm{x}$ & & & $\mathrm{x}$ & $\mathrm{x}$ & & & & \\
\hline Brevicoryne brassicae (Linnaeus, 1758) & $\mathrm{x}$ & & $\mathrm{x}$ & $\mathrm{x}$ & & & & $x$ & \\
\hline Capitophorus carduinus (Walker, 1850) & $\mathrm{x}$ & & & $\mathrm{x}$ & & & & & \\
\hline Capitophorus elaeagni (del Guercio, 1894) & & & & $\mathrm{x}$ & & & & & \\
\hline Capitophorus hippophaes (Walker, 1852) & $\mathrm{x}$ & & & $\mathrm{x}$ & $\mathrm{x}$ & & & & \\
\hline Capitophorus xanthii (Oestlund, 1886) & $\mathrm{x}$ & & & & & & & & \\
\hline Carolinaia caricis Wilson, 1911 & $\mathrm{x}$ & & & $\mathrm{x}$ & & & & & \\
\hline Carolinaia cyperi Ainslie, 1915 & $\mathrm{x}$ & & & $\mathrm{x}$ & $\mathrm{xx}$ & & & $\mathrm{x}$ & \\
\hline Cavariella aegopodii (Scopoli, 1763) & $\mathrm{x}$ & & & & $\mathrm{x}$ & & & & \\
\hline Cerataphis brasiliensis (Hempel, 1901) & $\mathrm{x}$ & $\mathrm{x}$ & $\mathrm{x}$ & $\mathrm{x}$ & $\mathrm{xx}$ & & $\mathrm{xx}$ & & $\mathrm{x}$ \\
\hline Cerataphis lataniae (Boisduval, 1867) & $\mathrm{x}$ & & $\mathrm{x}$ & & & & & & \\
\hline Cerataphis orchidearum (Westwood, 1879) & $\mathrm{x}$ & $\mathrm{x}$ & & $\mathrm{x}$ & $x x$ & & & & \\
\hline Chaetosiphon minor (Forbes, 1884) & & & & $\mathrm{x}$ & & & & & \\
\hline Chaitophorus minutus (Tissot, 1932) & $\mathrm{x}$ & & & & & & & & \\
\hline Cinara atlantica (Wilson, 1919) & $\mathrm{x}$ & & & & & & & & \\
\hline Cinara idahoensis Knowlton, 1935 & $\mathrm{x}$ & & & & & & & & \\
\hline Cinara louisianensis Boudreaux, 1949 & $\mathrm{x}$ & & & & & & & & \\
\hline Cinara pergandei (Wilson, 1919) & $\mathrm{x}$ & & & & & & & & \\
\hline Cinara tujafilina (del Guercio, 1909) & $\mathrm{x}$ & & & $\mathrm{x}$ & & & & & \\
\hline Coloradoa rufomaculata (Wilson, 1908) & $\mathrm{x}$ & & & & & & & & \\
\hline Dysaphis apiifolia (Theobald, 1923) & $\mathrm{x}$ & & & & & & & & \\
\hline Eriosoma lanigerum (Hausmann, 1802) & & & $\mathrm{x}$ & & & & & & \\
\hline Geopemphigus floccosus (Moreira, 1925) & $x$ & & & $\mathrm{x}$ & $\mathrm{x}$ & & & & \\
\hline Hyalomyzus eriobotryae (Tissot, 1935) & $x$ & & & & & & & & \\
\hline Hyalomyzus jussiaeae Smith, 1960 & $x$ & & & $\mathrm{x}$ & & & & & \\
\hline Hyperomyzus carduellinus (Theobald, 1915) & & & & & $\mathrm{xx}$ & & & & \\
\hline Hyperomyzus lactucae (Linnaeus, 1758) & $\mathrm{x}$ & & & $\mathrm{x}$ & $\mathrm{x}$ & & & & \\
\hline Hysteroneura setariae (Thomas, 1878) & $\mathrm{x}$ & & & $\mathrm{x}$ & $\mathrm{xx}$ & & & & \\
\hline Idiopterus nephrelepidis Davis, 1909 & $x$ & & & & & & & & \\
\hline Lipaphis erysimi (Kaltenbach, 1843) & $\mathrm{x}$ & & $x$ & $\mathrm{x}$ & $\mathrm{x}$ & $\mathrm{xx}$ & $\mathrm{x}$ & & \\
\hline Lizerius acunai (Holman, 1974) & $\mathrm{x}$ & & & & & & & & \\
\hline Macrosiphoniella sanborni (Gilette, 1908) & $\mathrm{x}$ & $\mathrm{x}$ & & & $\mathrm{xx}$ & & & & \\
\hline Macrosiphum euphorbiae (Thomas, 1878) & $\mathrm{x}$ & $\mathrm{x}$ & $\mathrm{x}$ & $x$ & $\mathrm{x}$ & & $\mathrm{x}$ & & \\
\hline Macrosiphum rosae (Linnaeus, 1758) & & & & $\mathrm{x}$ & & & & & \\
\hline Melanaphis sacchari (Zehntner, 1897) & & & $x$ & $\mathrm{x}$ & $\mathrm{xx}$ & $\mathrm{xx}$ & & & \\
\hline Microparsus brasiliensis (Moreira, 1925) & $\mathrm{x}$ & & $\mathrm{x}$ & $\mathrm{x}$ & $\mathrm{x}$ & & & & \\
\hline Microparsus olive Smith \& Tuatay, 1960 & $\mathrm{x}$ & & & $\mathrm{x}$ & & & & & \\
\hline Microparsus puertoricensis (Smith, 1970) & & & & $\mathrm{x}$ & & & & & \\
\hline Microparsus vignaphilus (E.E. Blanchard, 1922) & & & & $\mathrm{x}$ & & & & & \\
\hline
\end{tabular}




\begin{tabular}{|c|c|c|c|c|c|c|c|c|c|}
\hline Espèces & $\mathrm{Cu}$. & Ja. & Hi. & P.R. & Gu. & M.G. & Ma. & Bar. & Tr. \\
\hline Myzocallis discolor (Monell in Riley \& Monell, 1879) & $\mathrm{x}$ & & & & & & & & \\
\hline Myzus ornatus Laing, 1932 & $\mathrm{x}$ & & & $\mathrm{x}$ & $\mathrm{x}$ & & & & \\
\hline Myzus persicae (Sulzer, 1776) & $\mathrm{x}$ & & $\mathrm{x}$ & $\mathrm{x}$ & $\mathrm{x}$ & & $x$ & $\mathrm{x}$ & \\
\hline Pentalonia nigronervosa Coquerel, 1859 & $\mathrm{x}$ & & $x$ & $\mathrm{x}$ & $\mathrm{x}$ & $x x$ & $x$ & & \\
\hline Pleotrichophorus ambrosiae Hille Ris Lambers, 1969 & $\mathrm{x}$ & & & & & & & & \\
\hline Prociphilus erigeronensis (Thomas, 1879) & & & & $\mathrm{x}$ & & & & & \\
\hline Pseudoregma panicola (Tahashi, 1921) & $\mathrm{x}$ & & & $\mathrm{x}$ & $\mathrm{xx}$ & & & & \\
\hline Rhodobium porosum (Sanderson, 1900) & $\mathrm{x}$ & $\mathrm{x}$ & & $\mathrm{x}$ & $\mathrm{xx}$ & $\mathrm{xx}$ & & & \\
\hline Rhopalosiphonimus latysiphon (Davidson, 1912) & $\mathrm{x}$ & & & & & & & & \\
\hline Rhopalosiphum maidis (Fitch, 1856) & $\mathrm{x}$ & $\mathrm{x}$ & $\mathrm{x}$ & $x$ & $x$ & $\mathrm{xx}$ & $\mathrm{x}$ & $\mathrm{x}$ & \\
\hline Rhopalosiphum nymphaeae (Linnaeus, 1761) & $\mathrm{x}$ & & & $\mathrm{x}$ & $\mathrm{xx}$ & & $=$ & 1 & \\
\hline Rhopalosiphum padi (Linnaeus, 1758) & $\mathrm{x}$ & & & $\mathrm{x}$ & $\mathrm{x}$ & & & 2 & 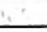 \\
\hline Rhopalosiphum rufiabdominale (Sasaki, 1899) & $\mathrm{x}$ & $\mathrm{x}$ & $\mathrm{x}$ & $\mathrm{x}$ & $\mathrm{x}$ & & & & \\
\hline Schizaphis gramimum (Rondani [1847] 1852) & $\mathrm{x}$ & & $\mathrm{x}$ & $x$ & $\mathrm{x}$ & & & & \\
\hline Sipha flava (Forbes, 1884) & $\mathrm{x}$ & & $x$ & $\mathrm{x}$ & $\mathrm{x}$ & & $\mathrm{x}$ & $x$ & $\mathrm{x}$ \\
\hline Sitobion avenae (Fabricius, 1775) & $\mathrm{x}$ & & & & $\mathrm{x}$ & & & & \\
\hline Sitobion cuscutae (Holman, 1974) & $\mathrm{x}$ & & & & & & & & \\
\hline Sitobion cyatheae (Holman, 1974) & $\mathrm{x}$ & & & & & 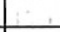 & & $\mathrm{A}$ & \\
\hline Sitobion lambersi David, 1956 & $\mathrm{x}$ & & & t & & & & & \\
\hline Sitobion luteum (Buckton, 1876) & $\mathrm{x}$ & & & $x$ & & & & & \\
\hline Sitobion martorelli (Smith, 1960) & $\mathrm{x}$ & & $\mathrm{x}$ & & & & & & \\
\hline Sitobion mesosphaeri (Tissot, 1934) & $\mathrm{x}$ & & & 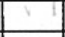 & $\mathrm{xx}$ & & & & \\
\hline Sitobion pauliani Remaudière, 1957 & $x$ & & - & 3 & & & 1 & & \\
\hline Sitobion ptericolens (Patch, 1919) & $x$ & & & 4 & & & 3 & & \\
\hline Sitobion salviae (Bartholomew, 1932) & $\mathrm{x}$ & & + & $x$ & & & & & \\
\hline Smynthurodes betae Westwood, 1849 & & & & $\mathrm{x}$ & & & & & \\
\hline Tetraneura nigriabdominalis (Sasaki, 1899) & $x$ & $\mathrm{x}$ & & $\mathrm{x}$ & $\mathrm{x}$ & & 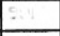 & & \\
\hline Tinocallis kahawaluokalani (Kirkaldy, 1907) & & & & $x$ & & & $T$ & & \\
\hline Toxoptera aurantii (Boyer de Fonscolombe, 1841) & $x$ & $x$ & $\mathrm{x}$ & $\mathrm{x}$ & $\mathrm{x}$ & & $\mathrm{x}$ & $x$ & $x$ \\
\hline Toxoptera citricida (Kirkaldy, 1907) & $\mathrm{x}$ & $\mathrm{x}$ & $\mathrm{x}$ & $\mathrm{x}$ & $x$ & $\mathrm{x}$ & $x$ & & $\mathrm{x}$ \\
\hline Tuberolachnus salignus (J.F. Gmelin, 1790) & $\mathrm{x}$ & & & & & & & & \\
\hline Uroleucon ambrosiae (Thomas, 1878) & $\mathrm{x}$ & & $\mathrm{x}$ & $\mathrm{x}$ & $\mathrm{xx}$ & & & & 2 \\
\hline Uroleucon erigeronense (Thomas, 1878) & $\mathrm{x}$ & & & $\mathrm{x}$ & $\mathrm{x}$ & & & & \\
\hline Uroleucon gravicorne (Patch, 1919) & $\mathrm{x}$ & & & $\mathrm{x}$ & & & & & \\
\hline Uroleucon pseudambrosiae (Olive, 1963) & $\mathrm{x}$ & & & & & & & & \\
\hline Uroleucon tuataiae (Olive, 1963) & $\mathrm{x}$ & & & & & & & & \\
\hline Uroleucon vernonicola (Holman, 1974) & $x$ & & & & & & & & \\
\hline Uroleucon zayasi (Holman, 1974) & $\mathrm{x}$ & & & & & & & & \\
\hline Utamphorophora commelinensis (Smith, 1960) & $\mathrm{x}$ & & & $\mathrm{x}$ & $\mathrm{x}$ & & & & \\
\hline Total espèces citées (94) & 82 & 15 & 24 & 56 & 41 & 10 & 14 & 10 & 8 \\
\hline
\end{tabular}

Tableau II. - Distribution des Aphides recensés dans les 11 îles antillaises où moins de 6 espèces ont été reconnues. $\mathbf{x}$ : espèces déjà citées dans la littérature; $\mathbf{x x}$ : espèces nouvellement observées; S.T.: Saint-Thomas; S.M.: SaintMartin, S.B. : Saint-Barthélemy, S.K. : Saint-Kitts; An.: Antigua; Mo. : Montserrat;Dé.: Désirade; A.S. : archipel des Saintes; S.L.: Sainte-Lucie; S.V.: Saint-Vincent; Gr.: Grenade.

\begin{tabular}{|l|c|c|c|c|c|c|c|c|c|c|c|}
\hline \multicolumn{1}{|c|}{ Espèces } & S.T. & S.M. & S.B. & S.K. & An. & Mo. & Dé. & A.S. & S.L. & S.V. & Gr. \\
\hline Aphis craccivora Koch, 1854 & & & $\mathrm{xx}$ & $\mathrm{x}$ & $\mathrm{x}$ & & $\mathrm{x}$ & & & $\mathrm{x}$ & \\
\hline Aphis gossypii Glover, 1877 & & $\mathrm{xx}$ & $\mathrm{xx}$ & & $\mathrm{x}$ & & & & & & \\
\hline Aphis nerii Boyer de Fonscolombe, 1841 & & $\mathrm{xx}$ & $\mathrm{xx}$ & & & & & & & & \\
\hline Aphis spiraecola Patch, 1914 & & & $\mathrm{xx}$ & & & & & & & & \\
\hline Cerataphis brasiliensis (Hempel, 1901) & & & & & & & $\mathrm{x}$ & & & & \\
\hline Cerataphis lataniae (Boisduval, 1867) & & & & & & $\mathrm{x}$ & & & $\mathrm{x}$ & & \\
\hline Microparsus brasiliensis (Moreira, 1925) & & & & & $\mathrm{x}$ & & & & & & \\
\hline Rhopalosiphum maidis (Fitch, 1856) & & & & & $\mathrm{x}$ & & & & & & \\
\hline Sipha flava (Forbes, 1884) & & & $\mathrm{x}$ & & & & & & $\mathrm{x}$ \\
\hline Toxoptera aurantii (Boyer de Fonscolombe, 1841) & $\mathrm{x}$ & & & & & $\mathrm{x}$ & & & & $\mathrm{x}$ & $\mathrm{x}$ \\
\hline Toxoptera citricida (Kirkaldy, 1907) & & $\mathrm{x}$ & & $\mathrm{x}$ & & & & $\mathrm{xx}$ & $\mathrm{x}$ & & \\
\hline Total espèces citées & $\mathbf{1}$ & $\mathbf{3}$ & $\mathbf{4}$ & $\mathbf{2}$ & $\mathbf{5}$ & $\mathbf{2}$ & $\mathbf{2}$ & $\mathbf{1}$ & $\mathbf{2}$ & $\mathbf{2}$ & $\mathbf{2}$ \\
\hline
\end{tabular}


semble assez largement répandue, elle a notamment été retrouvée au Brésil (Campinas, São Paulo) (REMAUDIĖRE, 1994) puis en Floride (HALBERT et al.). De même, Sitobion martorelli (SMITH, 1960 a); décrit de la République Dominicaine, était déjà signalé en Amérique centrale par SMITH \& CERMELI (1979). Deux des trois espèces décrites de Puerto Rico par SMITH (1960b) sont aussi présentes dans des pays de l'Amérique continentale: Acyrthosiphon bidenticola est mentionnée du Brésil et du Venezuela par SMITH \& CERMELI (1979), de Bolivie et du Mexique (REMAUDiĖRE et al., 1992) et est aussi connue de Panama (Remaudière, comm. pers.); Utamphorophora commelinensis est également citée du Brésil, du Venezuela et du Mexique, elle a été trouvé en outre au Pérou (Remaudière, comm. pers.).

Si l'on compare la diversité aphidienne de la Guadeloupe à celle de Cuba, on constate que les espèces cosmopolites + pantropicales représentent environ la moitié de la faune cubaine et les trois quarts de la faune guadeloupéenne; les espèces dont la distribution ne déborde pas le Nouveau Monde sont deux fois plus nombreuses à Cuba qu'en Guadeloupe. La relativement faible différence de latitude existant entre les deux îles $\left(23\right.$ et $17^{\circ} \mathrm{N}$.) n'explique pas cette situation; en revanche, leur position vis-à-vis des continents voisins semble justifier cette discordance : Cuba n'est qu'à $200 \mathrm{~km}$ de la Floride, c'est-à-dire de ce continent qui héberge près de 1500 espèces aphidiennes, alors que la Guadeloupe est à $2000 \mathrm{~km}$ de la Floride et à $600 \mathrm{~km}$ de l'Amérique du Sud, ce continent dont on connaît moins de 250 espèces de Pucerons (moins d'une centaine au Venezuela, le pays proche des Antilles).

Avec $36 \%$ d'espèces dont l'extension ne s'écarte pas de l'Amérique, Puerto Rico occupe une position intermédiaire entre Cuba (49\%) et la Guadeloupe (24\%).

REMERCIEMENTS. - L'auteur exprime sa profonde gratitude à son ami Georges Remaudière qui depuis de très nombreuses années lui a apporté son appui sans réserve et a identifié ou confirmé pour lui l'ensemble du matériel qu'il a collecté dans les Caraíbes. Sans ses conseils et son aide précieuse ce papier n'aurait certainement pas vu le jour. L'auteur exprime aussi ses remerciements à Mlle Gl. Boëcasse (LISP, Guadeloupe) et Mme M.A. Davidas (Fredon, Martinique) qui lui ont communiqué respectivement des spécimens de l'île de Marie-Galante et de la Martinique. Ilș remerciert également à l'INRA: MM. Jacques Fournet et Daniel Marival pour l'aide apportée dans l'identification des plantes-hôtes.

\section{AUTEURS CITÉS}

Aguilar J. d' \& BONFils J., 1962. - Problèmes entomologiques concernant la canne à sucre aux Antilles françaises. L'Agronomie tropicale, 1962 (7-8): 566-575.

ANONYMOUS, 1993. - Brown Citrus Aphid in Cuba. Caraphin News, 8: 8.

BENNETT F.D. \& ALAM M.M., 1985. - An annotated Check-List of the Insects and allied terrestrial Arthropods of Barbados. Caribbean Agricultural Research and Development Institute, $81 \mathrm{p}$.

BLANCHARD E.E., 1944. - Descripciones y anotaciones de afidoideos argentinos. Acta Zoologica Lilloana, Tucuman, 2 (1): 15-62.

BLANCHE D., 1956. - Maladies et ennemis de la canne à sucre aux Antilles françaises. Revue agricole sucrière et rhumière des Antilles françaises, 1 (1): 19-22.

DELPLANQUE A., 1976. - Insectes ravageurs des cultures maraîchères et vivrières aux Antilles françaises. Nouvelles Agronomiques des Antilles et de la Guyane, 2 (1): 22-47.

EASTOP V.F., 1961. - A study of the Aphididae (Homoptera) of West Africa. Trustees of the British Museum: $93 \mathrm{p}$.

EASTOP V.F. \& BLACKMAN R.L., 1988. - The identity of Aphis citricola van der Goot. Systematic Entomology, $13(2): 157-160$.

ÉTIENNE J., AUBERT B. \& LECLANT F., 1992. - Premier signalement de Toxoptera citricidus en Guadeloupe, Martinique, République Dominicaine et Sainte Lucie. Bulletin Phytosanitaire FAO, 40 (4): 162.

ÉtIENNE J., HuC A., BAYART J.D., DENON D. \& ANAIS G., 1994. - Toxoptera citricidus (Homoptera Aphididae) a vector of Citrus Tristeza Virus (CTV) in Guadeloupe; Distribution and Disease Investigation. 30 Congress CFCS, St Thomas, U.S.V.I., $30: 301-306$.

ÉTIENNE J., MARTINEZ M. \& ALAM M., 1993. - Toxoptera citricidus en Jamaïque : nouveau signalement pour les Caraïbes (Hom. Aphididae). Nouvelle Revue d'Entomologie (N.S.), 10 (4): 374.

FENNAH R.G., 1947. - The Insect Pests of Food-Crops in the Lesser Antilles. Department of Agriculture, Grenada \& Antigua : 209 p. 
FouRnET J., 2002. - Flore illustrée des phanérogames de Guadeloupe et de Martinique. Nouvelle édition. Cirad \& Gondwana éditions, 2 tomes : 2538 p.

HALBERT S.E. \& BROWN L.G., 1996. - Toxoptera citricida (Kirkaldy), brown citrus aphid identification, biology and management strategies. Floride Department of Agriculture and Consummer Services, Division of Plant Industry, Gainesville. Entomol., Circ. N $374: 6$ p.

HALBERT S.E., REMAUDIĖRE G. \& WEBB S.E., 2000. - Newly established and rarely collected aphids (Homoptera: Aphididae) in Florida and the southeastern United States. Florida Entomologist, 83 (1) : 79-91.

Holman J., 1974. - Los áfidos de Cuba. Instituto Cubano Del Libro, La Habana: 304 p.

LECLANT F., ÉTIENNE J. \& AuBERT B., 1992. - Alerte à la Tristeza: Toxoptera citricidus envahit l'arc Caraïbe. Phytoma. La défense des végétaux, $440: 32-34$.

MARTORELL L.F., 1975. - Annotated food plant catalog of the insects of Puerto Rico. Agricultural Experiment Station, University of Puerto Rico, Department of Entomology: $303 \mathrm{p}$.

Migliori A., Quiot J.B., LeClANT F., MARChOuX G. \& COLÉNO A., 1977. - Premières observations sur l'épidémiologie du virus de la Mosaïque du concombre et du virus de la Mosaïque de la Pastèque en Guadeloupe. Annales de Phytopathologie, 9 (2): 123-139.

NAUMANN-ETIENNE K. \& REMAudiĖRE G., 1995. - A commented preliminary checklist of the aphids (Homoptera: Aphididae) of Pakistan and their host plants. Parasitica, Gembloux, 51 (1): 3-61.

Remaudière G., 1994. - Revue et clé des espèces sud-américaines d'Aphidina et description d'un Aphis nouveau (Homoptera, Aphididae). Revue française d'Entomologie (N.S.), 16 (3):109-119.

REMAUDiėRE G. \& NAUMANN-ETIENNE K., 1991. - Découverte au Pakistan de l'hôte primaire de Rhopalosiphum maidis (Fitch) (Hom. Aphididae). Comptes rendus de l'Académie d'Agriculture de France, séance du 19 juin 1991, 77:61-62.

Remaudiėre G. \& Remaudière M., 1997. - Catalogue des Aphididae du Monde. Homoptera Aphidoidea. INRA Éditions, Paris (Techniques et Pratiques): 475 p.

REMAUDIÉRE G., SERTKAYA E. \& ÖZDEMIR I., 2003. - Alerte ! Découverte en Turquie du puceron américain Aphis illinoisensis nuisible à la vigne (Hemiptera, Aphididae). Revue française d'Entomologie (N.S.), $25(4): 170$.

Remaudière G., WeEmaels N. \& Nicolas J., 1992. - Contribution à la connaissance de la faune aphidienne de la Bolivie (Homoptera: Aphididae). Parasitica, Gembloux [1991], 47 (1): 19-46.

RUSSELL L.M., 1996. - Notes on Cerataphis brasiliensis and synonyms palmae, variabilis and fransseni (Homoptera: Aphididae) with a key to Cerataphis species living on palms and orchids. Proceedings of the entomological Society of Washington, 98: 439-449.

RYCKEWAERT P., 1998. - Guide de reconnaissance des insectes et acariens des cultures maraichères des Petites Antilles. Cirad/Flhor Martinique: $111 \mathrm{p}$.

SCHMUtTerer H., 1990. - Crop Pest in the Caribbean, with Particular Reference to the Dominican Republic. Deutsche Gesellschaft für technische Zusammenarbeit (GTZ) Gmbh, Eschborn: 640p.

Smith C.F., 1960 a. - Aphids (Aphidae: Homoptera) on "Cacao" in the Dominican Republic. Journal of Agriculture of University of Puerto Rico, 44:151-156.

1960 b. - New species of Aphidae: Homoptera from Puerto Rico. Journal of Agriculture of University of Puerto Rico, 44 : 157-162.

1970. - Notes on the genus Picturaphis and related genera with a new species of Picturaphis from Puerto Rico (Aphididae: Homoptera). The Journal of Agriculture of the University of Puerto Rico, 54 (4): 683-688.

SMith C.F. \& CERmeli M.M., 1979. -An annotated list of Aphididae (Homoptera)of the Caribbean Islands and South and Central America. North Carolina Technical Bulletin $N^{\circ} 259: 131 \mathrm{p}$.

SMith C.F., Martorell L.F. \& Perez Escolar M.E., 1963. - Aphididae of Puerto Rico. University of Puerto Rico. Agricultural Experiment Station, Technical Paper, 37: $121 \mathrm{p}$.

SMith C.F., MEdina GAud S., MARTORELl L.F. \& PEREZ EsCOlar M.E., 1971. - Additions and corrections to the Aphididae of Puerto Rico. The Journal of Agriculture of the University of Puerto Rico, $\mathbf{5 5}$ (2): 192-258.

Walker P.T., Waller J.M. \& Evans A.A.F., 1973. - The Insect Pests, Plant Diseases and Nematodes of Crops in Antigua, Ministry of Overseas Development, Technical Publications: $40 \mathrm{p}$.

WOODRUFF R.E., BECK B.M., SKELley P.E., SCHOTMAN C.Y.L. \& THOMAS M.C., 1998. - Checklist and bibliography of the insects of Grenada and the Grenadines. Center for Systematic Entomology, Memoir $\mathrm{N}^{\circ} 2: 286 \mathrm{p}$. 